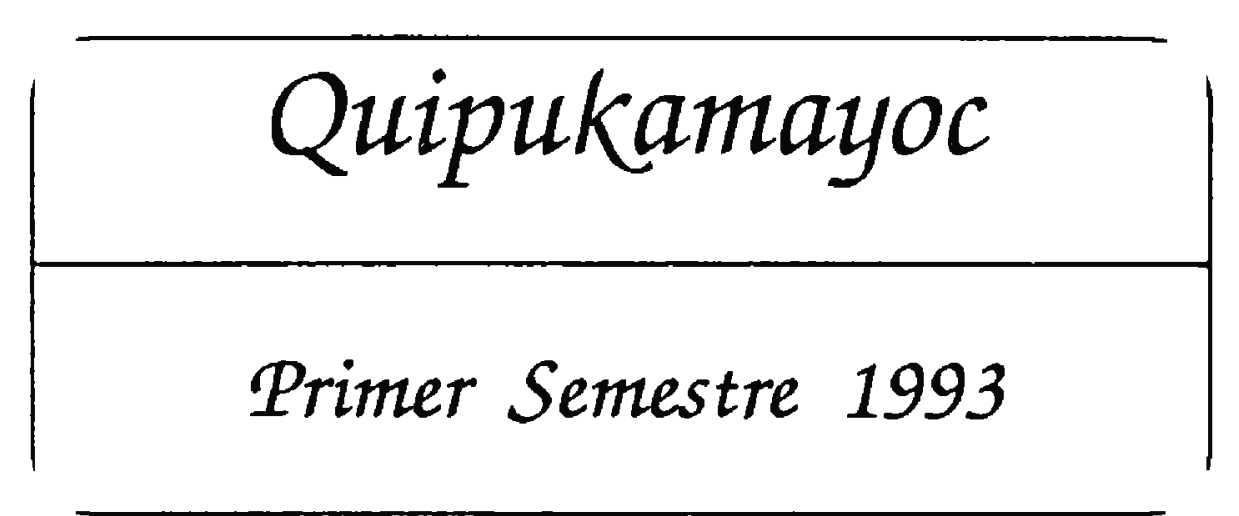

\section{"EL CONTADOR} ANTE LA

TRANSFORMACION SOCIOECONOMICA DE AMERICA LATINA"

\author{
Beltran Cova Velazquez
}

\begin{abstract}
Profesor de la Universidad Central de Venezuela..
\end{abstract}

Pues bien, les traigo un mensaje de fé, esperanza, optimismo, fraternidad, hermandad, deseo de trabajar en común por el bien de nuestros países en el presente, con un entroncamiento en nuestro pasado común y con visión de futuro. No podría ser de otra forma. Representamos QUINIENTOS (500) MILLONES de seres humanos, desperdigados en una extensa área geográfica, que partiendo de los Pirineos en Europa, se extiende entre los Oceános Atlántico y Pacífico, hasta la tierra del fuego. Un bloque humano caracterizado por: Una unidad linguística, histórica, religiosa, étnica: INDIOS AMERICANOS, IBEROS, ESPAÑOLES Y PORTUGUESES, NEGROS provenientes de varias zonas de Africa. Nuestros antepasados a los que rendimos nuestro recuerdo y homenaje sincero.

En nombre de la CONFEDERACION IBEROAMERICANA DE CONTADORES PUBLICOS SECCIONAL VENEZUELA, realizaré algunas reflexiones acerca del Tema general: “POR UN CONTADOR EFICIENTE COM-
PROMETIDO CON LA TRANSFORMACIONSOCIAL DE AMERICA LATINA" y específicamente, sobre "EL CONTADOR ANTE LA TRANSFORMACION SOCIOECONOMICA DE AMERICA LATINA".

El Contador Público colombiano Rafael Franco Ruiz, en su obra "REFLEXIONES CONTABLES", clasifica a los Contadores Públicos, en tres categorias, de acuerdo a su desempeño en cuanto a: ACTITUD ANTE LA PATRIA, ANTE LA FAMILIA, ANTE LA COMUNIDAD, ANTE LA PROFESION, ANTE SUS COLEGAS, ANTE EL TRABAJO, ANTE LA CIENCIA, ANTE LA POLITICA, ANTE EL ARTE, ANTE EL TRABAJO.

Estos tres tipos de profesionales son: LOS ARZOBISPOS, LOS BERBEOS, LOS GALANES. "LOS ARZOBIPOS": Son los contadores defensores de los monopolios transnacionales, representados por el INSTITUTO NACIONAL DE CONTADORES PUBLICOS, las grandes firmas de contadores (las 8 grandes) y un conjunto de colegas, unos beneficiarios de los dominadores y otros obnubilados por la producción técnica que ante la poca presencia de puntos de vista diferentes, queda como "verdad revelada" en el ambiente de la producción teórica. Se caracteriza este grupo por su instransigente defensa de las condiciones actuales del mercado profesional.

BERBEOS: Son los contadores con posición ambivalente (oportunistas) pues posan como nacionalistas, pero en su actividad de permanente coqueteo con los arzobispos, están prestos a mercadear hasta el último de sus principios. sus actitudes eclécticas los muestran apoyando los movimientos reivindicativos de los derechos de los contadores colombianos y al mismo tiempo respaldando organizaciones y eventos gremiales y técnicos de los arzobispos. Este grupo, podríamos decir no tiene principios, sólo le interesan los fines, los resultados del reparto.

GALANES: Es el grupo mayoritario o del montón, aquel sector que estáávido de justicia, tanto en la democracia laboral como en la vigilancia de los intereses económicos nacionales. Son los que defienden con autenticidad y con decisión los principios nacionalistas de la profesión".

Estamos hablando de tres enfoques vitales, intelectuales, de actuación de los contadores sobre el escenario que comprende la multipatria Ibcro-Luso-Centro-Sur América-del Caribe y Brasil. Con problemas específicos en cada región o grupo de países, pero que desde el 12 de octubre de 1942, van a formar una simbiosis que nos ha unido en el devenir histórico. España, ha pasado por diferentes formas de gobicrnos, ha encarado y sobrellevado monarquías, república, gobierno socialista, guerras, revoluciones, y, hoy muestra un adelanto extraordiario que le ha permitido ingresar a la Comunidad Económica Europea. En el Campo de la Ciencia de la Contabilidad ha realizado importantes avances y aportes, en el seno de la UNION EUROPEA DE EXPERTOS CONTABLES, $y$, han compartido con los colegas hispano-americanos y brasileños los logros alcanzados. Varios de sus máximos representantes han viajado permanentemente a nuestros países. Los CONTADORES portugueses también han hecho investigaciones y formulaciones teóricas aplicadas a su realidad.

Ahora bien, hablaremos de América; desde México hasta 
la Patagonia, región que ha sufrido las embestidas de: Losconquistadores, colonizadores, neo-colonizadores, imperialistas y proto-imperialistas, que la han convertido desde el pasado remoto en región explotable en cuanto a: sus masas humanas, recursos naturales, sus riquezas, sus tierras. Adonde ha podido llegar el visitante ajeno a nuestros suelos, ha habido: Linchamientos, matanzas en masa, saqueo, robo, destrucción, imposiciones, esta ha sido la constante a través de la historia y los experimentos que han hecho hombres y mujeres notables de nuestros paises han terminado en invasiones, cuartelazos, intervenciones exteriores. A través de este lapso histórico de quinientos (500) años, sólo un experimento de tipo nuevo se ha podido mantener y es el de la CUBA SOCIALISTA, donde su pueblo y su gobierno están dispuestos a repetir las gestas de Esparta y de Numancia, pero haciendo uso de los avances tecnológicos de la ciencia militar moderna, también se hará sentir en los bosques, en las universidades, en los templos, en las fábricas, en las sedes de los gobiernos, el ruido infernal de las bombas al explotar: Cuba no está sola, los galanes de toda la humanidad unirán esfuerzos para darle su apoyo, $y$, repetiremos con su pueblo y sus líderes ¡PATRIA O MUERTE VENCEREMOS!.

El escenario del Caribe caracterizado por el escritor colombiano Germán Arciniegas en su libro ENTRE LA LIBERTAD Y EL MIEDO “.... ésta no es ni América Latina, ni Española; es América Caribe. Están revuel tas colonias, semicolonias, protectoras, repúblicas, pseudorepúblicas, democracias y antidemocracias. Del lado de la América Central, fuera de México, hay seis estados independientes, una faja de tierra de los Estados Unidos y una colonia inglesa disputada por Guatemala. al frente, un archipiélago endemoniado. Curazao pertenece a la reina Juliana, y en la capital se habla papiamento. En Martinica que es de Francia -se habla creole. Hay unas islas que los daneses vendieron a los Estados Unidos, y aún se llaman vírgenes. En Trinidad, que está bajo la corona de Inglaterra, hay templos de hindúes y sinagogas: se llama las ciudades de América. Las ciudades de Haití parecen traídas de Africa. Puerto Rico es la isla española que se ha quedado en la mitad del camino de su independencia. Venezuela tiene islas, y las tiene Colombia, y hay Islas de Honduras arrendadas a Estados Unidos. La Isla de Santo Domingo está partida en dos. La mitad es la república negra de Haití, la otra mitad, que guarda toda la tradición española del descubrimiento, ha perdido hasta el nombre de su Capital. .."

Desde que el escritor Arciniegas publicó este libro, hasta la fecha ha habido cambios profundos en algunos países del Caribe, como en: CUBA REPUBLICA DOMINICANA, JAMAICA, GUYANA, SURINAM, BARBADOS, CURAZAO, TRINIDAD-TOBAGO.

Los contadores comprometidos con la transformación socioeconómica de América Latina, debemos utilizar para medir el avance de nuestras sociedades, LA MATRIZ DE CONTABILIDAD SOCIAL (MCS), “... que ha sido diseñada para analizar de una manera coherente la distribución del ingreso y sus diversas modalidades, es decir, tanto factoriales como instituciones. En conseucencia, este instrumento analítico está en capacidad de responder a la pregunta: ¿Quién obtiene el Producto Nacional Bruto?. Y además, nos permite disponer de cierta evidencia estadística sobre las condiciones de vida en el país, siempre que estas puedan ser medidas por el consumo de bienes y servicios para satisfacer las necesidades humanas", LINO A. CLEMENTE "La distribución del ingreso en la matriz de contabilidad social. La experiencia venezolana en 1982 y 1984 ". El autor establece 4 estratos que agrupan a las familias, atendiendo al ingreso promedio mensual de los hogares. Los estratos se han denominado, en orden ascendente de acuerdo al ingreso. De manera que:

Estrato I corresponde entre Bs. $0.00 \mathrm{y}$

$3,000.00$

Estrato II corresponde entre Bs.

$5,000.00$

Estrato III corresponde entre Bs. $3,001.00$ y Bs. $7,000.00$

Estrato IV corresponde entre Bs.

$5,001.00$ y Bs.

Nota $1 \$$ USA $=$ Bs. 4.30

Del análisis de los cuadros podemos concluir que:

1 - Para 1982, el 71,09 de las familias venezolanas, obtendrían un ingreso, equivalente a:

$45,61=22,67$ nivel de ingresos entre 0.00 y $3,000.00$. $25,46=24,09$ nivel de ingresos entre -3001 y -5000.00 .

71,07

Esto es, el 71,07 del total de las familias venezolanas, obtuvieron ingresos entre 3,000 y 5,000.00, es decir entre 679,67 y $1,162.78$ US\$.

2 - Según el cuadro siguiente, para 1984, la situación fue:

$41,93=19,96$ nivel de ingreso entre $0.003,000.00$.

$24,49=22,02$ nivel de ingreso entre $3,001.00$ y $5,00.00$

66,42 .

Vemos que el índice varia de 71,07 a 66,41 , lo que equivale a un descenso del $4,65 \%$. Es decir un número mayor de familias pasó a engrosar los estratos III y IV de mayores ingresos.

El estudio de este índice de la distribución del ingreso, es muy importante, para la toma de decisiones en cuanto a política general de las naciones modernas ya, que en síntesis las luchas por obtener una mayor parte del ingreso nacional, siempre animará a los seres humanos.

Para profundizar en el papel que deben asumir los contadores ante el desarrollo y la transformación socieconómica de América Latina, es conveniente citar lo anotado al respecto por el colega contador público, economista, investigador y catedrático en varias universidades de Colombia. Dr. JULIO SILVA COLMENARES, en Su obra "TRAS LA MASCARA, DEL SUBDESARROLLO: DEPENDENCIA Y MONOPOLIO". . . La principal clase explotada en América Latina y El' Caribe es el proletariado, cuya conciencia política y grado de| organización sindical se elevan. Su contingente pasa actual- 


\section{DISTRIBUCION DEL INGRESO EN VENEZUELA POR ESTRATOS \\ DE INGRESO SALARIAL}

(Porcentajes)

1982

\begin{tabular}{ccccc}
\hline & POBLACION & INGRESO & POB. AC. & ING. AC. \\
I & 45,61 & & & \\
II & 25,46 & 22,67 & 45,61 & 22,66 \\
III & 13,30 & 24,09 & 71,07 & 46,76 \\
IV & 15,63 & 18,04 & 84,37 & 64,80 \\
& & 35,20 & 100,00 & 100,00
\end{tabular}

1984

\begin{tabular}{lrrrr}
\hline ESTRATO I & 41,93 & 19,96 & 41,93 & 19,96 \\
ESTRATO II & 24,49 & 22,02 & 66,42 & 41,98 \\
ESTRATO III & 13,38 & 16,67 & 79,80 & 58,65 \\
ESTRATO IV & 20,20 & 41,35 & 100,00 & 100,00
\end{tabular}

LA DISTRIBUCION DEL INGRESO EN VENEZUELA POR ESTRATOS DE INGRESO TOTAL

1982

\begin{tabular}{lllrr}
\hline & POBLACION & INGRESO & POB. AC & ING. AC \\
ESTRATO I & & & & \\
ESTRATO II & 45,61 & 12,24 & 45,61 & 12,24 \\
ESTRATO III & 25,46 & 16,98 & 71,07 & 29,29 \\
ESTRATO IV & 13,30 & 32,27 & 84,37 & 61,49 \\
& 15,64 & 38,51 & 100,00 & 100,00
\end{tabular}

1984

\begin{tabular}{lrrrr}
\hline ESTRATO I & 41,93 & 10,96 & 41,93 & 10,06 \\
ESTRATO II & 24,49 & 15,85 & 66,42 & 25,91 \\
ESTRATO III & 13,38 & 24,74 & 79,80 & 50,65 \\
ESTRATO IV & 20,20 & 49,35 & 100,00 & 100,00
\end{tabular}

mente de 40 milones, es decir, la décima parte de todos los obreros del mundo capitalista. Parte apreciable de este contingente trabaja para las transnacionales, que ocupan no menos de cinco millones de personas. El avance del capitalismo contribuye objetivamente al aumento y la toma de conciencia del proletariado, aportando otro de nuestros rasgos característicos, así no hayamos tenido un pleno desarrollo del capitalismo en el sentido clásico, la respuesta al atraso se relaciona intimamente con la solución de la contradicción trabajo- capital, esto es, la alternativa de la dependencia no es el desarrollo capitalista hasta llegar a los niveles altos, sino la revolución de liberación nacional y cambio social, antiimperialista y antioligárquica, democrática y popular, que abre el camino al socialismo. Es obvio que la estructura clasista descrita en los párrafos anteriores no es un molde que puede aplicarse automáticamente a cualquier país. La correlación de poder entre las clases y su composición numérica varía, pero sí podemos verla como un panorama general de América 
Latina y el Caribe, con excepción de Cuba, aplicable especialmente a los países de mayor desarrollo relativo. Esta estructura, producto de una economía deformada, es correlativa a una desastrosa distribución de ingreso:

\section{INGRESO TOTAL-DISTRIBUCION}

$\%$

$\begin{array}{r}20 \\ 30 \\ 30 \\ 15 \\ 5 \\ \hline\end{array}$

100
DETALLE

\% RECIBE

\begin{tabular}{lr} 
Pobre & 3 \\
Sector Menos pobre & 10 \\
Ingresos Apreciables & 24 \\
Altos Ingresos & 29 \\
Cúspide & 34 \\
\cline { 2 - 2 } & 100
\end{tabular}

Como señala la CEPAL, no menos de 150 millones de latinoamericanos tienen que sobrevivir con un ingreso per capita anual inferior a US\$ 180 (dólares de 1972), con el agravante de que el ingreso se concentra más con el avance del capitalismo, en lugar de redistribuirse.

Como ven los colegas, el trasfondo de la situación económico-social en que vivimos es bastante cruel, y, se requerirá un gran esfuerzo de todos para que estos índices alarmantes se puedan superar, en beneficios de las grandes mayorías de nuestros países.

La situación en lo atinente a ciertos aspectos de salud y a condiciones generales de vida de los países del Caribe, los hemos obtenido, siguiendo la rientación del Dr. GUAROCUYA BATISTA DEL VILLAR, Ex-Rector, Director de la División de Estudios de Postgrado y Educación Permanente, de la Universidad Autónoma de Santo Domingo, denominada "LA CRISIS DEL MODELO MEDICO HEGEMONICO DOMINICANO. ALTERANTIVAS PARA EL AÑO 2,001". El basa su estudio, sobre 4 categorías básicas, SALUD, ATENCION PRIMARIA, MODELO MEDICO, HEGEMONIA.

Salud, según "Sergio Arouca. .., constituye un espacio para la hegemonía de las clases sociales, a través de políticas relacionados con la reproducción social" y la Organización Mundial de la Salud, que considera la salud no sólo como la auscncia de enfermedad, sino también como un estado de bienestar biológico, psicológico y social". La categoría HEGEMONIA, queda definida en la siguiente forma "con esta categoría, de uso común en los lenguajes sociales, debe las teorizaciones al italiano Antonio Gramci, nos referimos a la capacidad de un grupo o clase social, para presentar un intere como si fuera el interés común y natural de la sociedad global. Aún más, hegemonía significará la habilidad de los mencionados sectores para obtener la aceptación de esos intereses minoritarios por parte de la mayoría de las personas, sin el uso de la coerción y, por consiguiente, mediante el consenso. Es una forma de síntesis de la dominación, pero en el terreno ideologico. Muy pertinente para la hegemonía es el funcionamicnto de los llamados "APARATOS IDEOLOGICOS", escuela, iglesias, estructuras de salud, partidos políticos y otros, todos pertenecientes a la "sociedad civil". para el año 1981, esta era la situación de Salud en ra Repúbl : Dominicana, según los indiadores clásicos:

- $\quad$ 20-40 \% de la población total sin atención en salud (estructuras organizadas).

Sitema Nacional de Salud: Inexistente.

$65 \%$ de los niños menors de 5 años con desnutrición proteico-calórica.

- Mortalidad infantil, 130.5 por mil nacidos vivos.

- Primera causa de muerte: trastornos gastro-instestinales de los lactantes.

Sólo $12.7 \%$ de la población económicamente activa está "protegida" por el Instituto de Seguro Social. lo que quiera decir. 2,600.00 personas no protegidas.

- Número de habitantes por Médico 2,300.

El Dr. Guarocuya, trata du explicar el origen de los resultados del cuadro general anterior, por medio de las TRES TESIS:

TESIS 1: EL MODELO MEDICO DE LA REPUBLICA DOMIICANA ESTA SUMIDO EN UNA PROFUNDA CRISIS.

TESIS 2: LA CRISIS DEL MODELO MEDICO ES UNA COMPONENTE DE LA CRISIS DE LA SOCIEDAD HEGEMONICA.

TESIS 3: LA CRISIS DE LA SOCIEDAD DOMINICANA ES LA CONSECUENCIA DE LA INTERVENCION MILITAR NORTEAMERIANA DE 1965.

El Dr. Bautista del Villar, cita una serie de aspectos que fundamentan su exposición: Indices de calidad de vida material, desempleo, crecimiento económico, educación, vivienda, costo de la vida, nivel de los salarios, tasa medida bruta de las ganancias de los capitalistas: $1964=57 \%, 1965=49$ $\%, 1966=80 \%, 1967=91 \% 1968=100 \%, 1969=132$ $\%, 1970=164 \%, 1971=197 \%$.

Como se ve, la tasa de intereses cobradas por los capitalistas es ua elemento que influye negativamente en la dinamización de la economía. Otros elementos, que complementan el cuadro general pintado por el autor son: El control de la natalidad, priorización de la medicina privada bajo los auspicios de las entidades financieras privadas, suscribo totalmente la conclusión general de Batista del Villar de que "El derecho del pueblo a una buena salud, significa la soberanía permanente del pueblo. Todos los datos sugieren, que sin una transformación radical de las estructuras económicas, sociales y políticas, de las sociedades subdesarrolladas, las crisis de sus modclos médicos hegemónicos no será supcrada, porque esta crisis es un componente de la crisis social global. Si csta transformación ocurre, entonces con puntualidad, pero no antes, el pucblo se organizará y se movilizará para mejorar sus índices de salud. Tenemos algunos ejemplos en la hisoria reciente de Amćrica Latina y El Caribe.

Pienso que algunos sectores políticos, económicos, sociales, que apoyan a los contadores Arzobispos, se han planteado que para superar todos los males reseñados y otros más que aquejan a la sociedad del SIGLO XX, se pucden resolver fijando espectativas por cientos de años, milenios, cras geo- 
Das. Lamentablemente esto no es posible, y los pueblos J siben tomar en sus manos junto con las galanas y los galanes b solución de sus propios problemas y, tener en cuenta que unto con sus enemigos declarados, los imperialistas, los atifundistas, los empresarios retrógados, los científicos linres de todas leyes, deben colocar en primer plano, cuando la ocasión se presente a los contadores arzobispos y a sus colanoradores inmediatos.

\section{ACCIONES PRACTICAS:}

I.- Desalienación: Filosófica, religiosa, científica, cultural, tccnológica, empresarial, artística, deportiva, sanitarias, de invenciones tecnológicas, de altas tecnologías, sanitarias, de viviendas, de pro-acción de alimentos agropecuarios, educativa:

- Hacen lo nuestro con nuestro propio barro.

- No sea espejo para reflejar lo que hacen en otros países.

- Defensa irrestricta de nuestras culturas tradicionales, de los recursos naturales de nuestros países.

- Colaborar en el rormal suministro de tecnologías apropiadas en nuestra profesión a los gobiemos a los efectos de que los recargos que administran, sean hecho con eficiencia y honestidad para que la sociedad y especialmente en el "habitad" de los sectores más pobres de nuestros países.

- Aplicar la matriz de contabilidad social para medir el efecto del gasto $\mathrm{I}$ ubico en países nuestros.

- Propender a mejorar la calidad de la educación para la formación de los contadores públicos.

- La valorización de la profesión del contador público, mediante la lucha para lograr la aprobación de la ley de ejercicio profesional, nacionalistas y modernas.

- La sindicalización de los contadores púbicos, una palabra para la defensa de las codiciones de trabajo en cuanto a: represcntatividad, compensaciones salariales, de ascensos, de desarrollo intelectual y científicas. Las condic iones de trabajo de los jóvenes, las mujeres, los colegas veteranos, originados, asociados.

- La racionalización del trabajo de los contadores públicos: Del día de 24 horas al de 60 horas y todavía a los colegas les faltan horas para super contratarlas.

Que alineación!

- Hag: una humanización del entorno de la profesión!

- Todos contratados!

- Tiburones, caribes, pecho colorados!

II.- Haga un impulso de los requerimientos mínimos para el funcionamiento de una Escuela, Facultad de Contaduría Pública en Ibẹroamérica, Brasil, Puerto Rico.

A. Unas bibliotecas con publicaciones especializadas, y períodicas.

B. Un Instituto de Investigaciones en el que haya profesores investigadores a dedicación exclusiva que se dediquen a la actualización de los programas de estudio a nivel de Prc-Grado, Post-Grado, Maestría, Doctorado. Que di- señe cursos de extensión, seminarios, foros, estudios de situaciones especiales de los municipios, los estados, las naciones $o$ industrias específicas.

C. Un Laboratorio de Computación o Centro de Cómputo donde los estudiantes docentes, empleados, ciudadanos ajenos a la universidad, empresas, organismos oficiales, tengan un elemento válido para estudiar, y procesar la información a través de la informática.

D. Un Laboratorio de Idiomas, donde se estudien los idiomas: Portugués, Francés, Alemán, Inglés (de USA y de Inglaterra).

Nota: Debe ser exigencia que los Licenciados para poderse graduar acrediten el conocimiento del portugués y de cualquier otro idioma.

E. Un Centro Coordinador de Pasantías para que se puedan canalizar las permanencias obligatorias en los organismos del Estado por un mínimo de dos (2) años como requisito indispensable para poder graduarse. Además de presentar una monografía en la que resuma la experiencia adquirida.

F. Un Departamento de Orientación para los graduandos, que los sitúe en los ambientes de:

1. La investigación científica con dedicación a la docencia.

2. El ejercicio como Contador Público independiente, bien como:

2.a Por cuenta propia, creación de su propia firma.

2.b Como asesor o consultor en las diferentes especialidades de la profesión.

2.c Contratado, empleado a tiempo completo en una empresa o firma de Contadores Públicos.

2.d Funcionarios (Carrera de Alta Gerencias en los organismos del Estado).

G. Organizar Seminarios de Alto Liderazgo en los que se estimule a los graduandos a alzar la vista. A elevar las miras y aspirar a ser: Presidentes de Repúblicas, Presidentes de Empresas. Grandes Lideres: Científicos, Empresarios Educadores, pero sobre todo: ¡Qué sean mujeres y hombres con gran sensibilidad social!

\section{III.-El Contador eficien te comprometido con la transfor-} mación social de América Latina debe:

1ro.) Fijar pautas para que la cancelación de la deuda externa e interna no signifique el estancamiento y la pauperación de nucstros países. Se deben establecer límites razonables para su cancelación. "Paguemos la deuda ecológica y no la deuda externa para que desaparezca el hambre y no el hombre "Como lo dijo Fidel Castro, en la Cumbre de la Ticrra, Río de Janciro, Brasil, Junio 12 de 1992 (Anexo 1).

2do.) Para lograr una renegociación permanente para la impagable deuda de nuestros países, se deben impulsar proyectos para "El canje de la deuda por conservación".

Aprovechar las experiencias que han tenido sobre el par- 
ticular: Bolivia, Ecuador, Costa Rica, que mediante proyectos concretos han logrado reducir sus deudas externas en sumas significativas.

3ro.) Participar en estudios multidisciplinarios en sus países para buscar establecer unos términos de intercambio en el mercado internacional, que privilegie el precio de las materias primas que exportamos y facilite la adquisición de patentes de inversión, tecnologías, adelantos científicos y bienes suministrados por los países altamente desarrollados.

4to.) Los contadores deben participar activamente en la celebración de los tratados comerciales y dar su opinión sobre la justeza de los mismos. Señalar los elementos inconvenientes para los países.

5to.) Los contadores deben hacer causa común con las personalidades nacionalistas y con estas para trabajar contra los vicios derivados de la corrupción administrativa. El saqueo mediante contratos leoninos y la entrega de nuestros países a la voracidad de los países imperialistas.

6to.) Deben también unir esfuerzos para detener la acción de las empresas transnacionales de Auditoría y Consultoría en general, que embilece la profesión, como restan posibilidades de trabajo a los Contadores Nacionales y actúan en contra de los intereses nacionales y en beneficio de los empresarios o países extranjeros que los contratan.

7mo.) Los contadores están en el deber de actualizar, mediante un estudio profundo de sus países un modelo de informe del auditor externo nacionalista

8vo.) Los contadores deben trabajar por lograr la colegiación única para poder impulsar la profesión. (Anexo II).

9no.) Los contadores deben prestar su colaboración para la elaboración del código Iberoamericano de Contabilidad y Auditoría, (Anexo III).

10mo.) Los contadores deben prestar su colaboración para fomentar y apoyar a la Confederación Iberoamericana de Contadores Públicos, de acuerdo a sus objetivos, contenidos en el artículo 6 de sus estatutos. (Anexo IV).

11ava.) Ayer fueron República Dominicana, después: Guatemala, México, Nicaragua, Argentina (caso Las Malvinas), recientemente Panamá, hoy -hace treinta años- lo es la República hermana de Cuba, víctima de la intervención inicua del gobierno de los Estados Unidos de Norteamérica, por la aprobación de la Ley Torricelli, el 23/10/92. El consejo Universitario de la Universidad Centro Occidental Lisandro Alvarado, de la Ciudad de Barquisímetro, de la República de Venezuela, aprobó en su sesión ordinaria $N^{2} 555$, un comunicado de solidaridad.

Tomando en consideración que este asunto es de vital importancia para el futuro de la humanidad, pido muy respetuosamente que esta VI Conferencia de Facultades y Escuelas de Contaduría Pública de América Latina, emita un acuerdo solidario con el pueblo y el gobierno de la República de Cuba y condene la acción del gobierno de los Estados Unidos de Norteamérica, por ser antijurídico, improcedente y desconsiderado.

\section{Anexo I}

Fidel Castro en la Cumbre de la Tierra

\section{"PAGUEMOS LA DEUDA ECOLOGICA Y NO LA DEUDA EXTERNA PARA QUE DESAPAREZCA EL HAMBRE Y NO EL HOMBRE"}

Una importante especie biológica está en riesgo de desaparecer por la rápida y progresiva liquidación de sus condiciones naturales de vida: EL HOMBRE.

Ahora tomamos conciencia de este problema cuando casi es tarde para impedirlo. Es necesario señalar que las sociedades de cosumo son las responsables fundamentales de la atroz destrucción del medio ambiente. Ellas nacieron de las antiguas metrópolis coloniales y de políticas imperiales que a su vez engendraron el atraso y la pobreza que ya azotan a la mayoría de la comunidad.

Con solo el $20 \%$ de la población mundial ellas consumen las dos terceras partes de los metales, las tres cuartas partes de la energía que se produce en el mundo.

Han envenenado los mares y rlos, han contaminado el aire, han debilitado y perforado la capa de ozono, han saturado la atmósfera de gases que alteran las condiciones climáticas con efectos catastroficos que ya empezamos a padecer.

Los bosques desaparecen, los desiertos se extienden, miles de millones de toneladas de tierra fértil van a parar cada año al mar, numerosas especies se extinguen, la presión poblacional y la pobreza conducen a esfuerzos desesperados para sobrevivir aún a costa de la naturaleza.

No es posible culpar de esto a los países del Tercer Mundo, colonias de ayer, naciones explotadas y saqueadas hoy por un orden económico mundial injusto. La solución no puede impedir el desarrollo a los que más lo necesitan, lo real es que todo lo que contribuya al subdesarrollo y la pobreza, constituye una violación flagrante de la ecología.

Decenas de millones de hombres, mujeres y niños mueren a cada año a consecuencia de esto, más que en cada una de las guerras mundiales. El intercambio desigual, el proteccionismo y la deuda externa agreden la ecología y propician la destrucción el medio ambiente.

Si se quiere salvar la humanidad de esa autodestrucción, hay que distribuir mejor las riquezas y las tecnologías disponibles en el planeta, menos lujos y menos despilfarro en unos pocos paises para que haya menos pobreza y menos hambre en gran parte de la tierra.

Nomás transferencias al Tercer Mundo de estilos de vida y hábitos de consumo que arruinan el medio ambiente. Hagase más racional la vida humana

Apliquese un orden económico internacional justo. Utilice toda la ciencia necesaria para el desarrollo sostenido sin contaminación.

Paguese la deuda ecológica y no la deuda externa. 
Desaparezca el hambre y no el hombre.

Cuando las suuestas amenazas del comunismo han desaparecido y no quedan y a pretexto para guerras frías, carreras armamentistas y gastos militares. Que es lo que impide dedicar de inmediato esos recursos a promover el desarrollo del Tercer Mundo y combatir la amenaza de destrucción ecológica del planeta?

Cesen los egoísmos.

Cesen los hegemonismos.

Cesen la insensibilidad, la irresponsabilidad y el engaño mañana sera demasiado tarde para hacer lo que debimos haber hecho hace mucho tiempo.

Gracias.

Río de Janeiro, junio 12 de 1992.

\section{Anexo II \\ SOBRE LA COLEGIACION DEL CONTADOR}

LA CONFEDERACION IBEROAMERICANA DE CONTADORES PUBLICOS INTERPRETANDO LA LETRA Y ESPIRITU DE SUS OBJETIVOS FUNDAMENTALES QUE ESTABLECEN:

a. VELAR POR EL RESPETO DEL CONTADOR PUBLICO EN EL EJERCICIO DE SU PROFESION.

b. TENDER A QUE EL CONTADOR PUBLICO ADQUIERA UNA ADECUADA CONCIENCIA PROFESIONAL Y SOCIAL ACORDE CON SU PARTICIPACION INTEGRAL EN EL DESARROLLO DE LA COMUNIDAD.

c. PROPUGNAR HASTA LOGRAR QUE EL CONTADOR PUBLICO EN LOS PAISES CONFEDERADOS SELEOTORGUE EL RECONOCIMIENTO A SUCAPACIDAD PROFESIONAL.

IDENTIFICA LA COLEGIACION DEL CONTADOR PUBLICO COMO EL INSTRUMENTO JURIDICO-POLITICO MAS EFECTIVO PARA VIABILIZAR Y CONCRETAR O CONSOLIDAR, SEGUN SEA EL CASO, LOS PROPOSITOS ARRIBA SEÑALADOS.

EN ESTE SENTIDO, LA CONFEDERACION IBEROAMERICANA DE CONTADORES PUBLICOS RATIFICA EL OFRECIMIENTO DE SU MAS DECIDIDO APOYO A TODOS LOS GREMIOS CONFEDERADOS QUE HOY LUCHAN POR OBTENER, EN SUS RESPECTIVOS PAISES, LA COLEGIACION OBLIGATORIA DE LOS PROFESIONALES DE LA CONTABILIDAD.

\section{Anexo III \\ "CODIGO IBEROAMERICANO \\ DE CONTABILIDAD \\ Y AUDITORIA" \\ (Proyecto de Investigación)}

\section{INTRODUCCION}

La economía atraviesa actualmente un momento histórico fundamental al estar conformándose en el mundo un nuevo orden económico internacional. En este nuevo marco económico supranacional adquiere especial importancia la economía de libre mercado, la cual después de la revolución acaecida en los países del Este, ha pasado ya a contar con una general aceptación en este nuevo orden mundial. Ahora bien, esta general aceptación se mantendrá siempre supeditada a que en los modelos económicos se cumplan las reglas de competencia leal, se cuente con unos ciertos niveles de cobertura social y se asuma un elemental principio de transparencia en la información de aquellas unidades económicas que protagonizan el discurrir del sistema macroeconómico global.

En este ámbito cobra así una especial trascendencia la información económica referente a la unidad motor del sistema de libre mercado: la unidad empresarial. Esta información económica, elemento fundamental de transparencia en este contexto, se instrumenta a través de la Contabilidad.

Es importante recordar el hecho de que la calidad de la información tiene una directa influencia en la eficiencia de las decisiones y que el carácter público de los estados contables viene a justificar la necesidad de su auditoría por profesionales cualificados

Actualmente, la información contable está experimentando un fuerte y continuado proceso de normalización internacional, principalmente entre los denominados países desarrollados. Ese proceso exige un punto de partida en la descripción de las normas, su sistematización y su análisis, a fin de otorgar la base material de una futura consolidación y armonización global.

En este contexto de la información contable la necesaria caracterización de la comunidad iberoamericana toma rango de protagonismo con una fecha ya mítica antes de su acaecimiento: 1992. En ese año, en el que se cumple el quinto centenario del Descubrimiento de América, va a tener una proyección especial todo lo relacionado con dicha comunidad, convirtiéndose asi en la dicha idónea para la publicación de una obra integral como este "CODIGO IBEROAMERICANO DE CONTABILIDAD Y AUDITORIA".

Dado el innegable papel armonizador de la información que cumplen las normas contables y auditoras, es impensable en este contexto un mundo centro sin un espacio con personalidad propia para el colectivo iberoamericano. 


\section{OBJETIVOS DEL PROYECTO: "CODIGO IBEROAMERICANO DE CONTABILI. DAD Y AUDITORIA"}

\section{NECESIDAD DEL PROYECTO:}

Un proyecto de investigación que se estructure sobre la temática, social, económica y cultural de Iberoamérica lleva en sí independientemente del área específica que se aborde una necesidad política: la de colaborar en el desarrollo de la comunidad iberoamericana la de otorgar una base material para su consolidación armónica.

En el caso concreto de este proyecto, reunir en un único texto la normativa vigente en un área tan trascendental para la economía como la contabilidad, donde se incluyan la totalidad de los países de la comunidad iberoamericana, satisface múltiples objetivos y cubre diversas necesidades, tanto desde una perspectiva macroeconómica (necesidad de información útil viable para procesos de toma de decisiones que abarcan nuevos espacios económicos), como, bajo una proyección más específica, satisfaciendo necesidades concretas de profesionales, empresarios, inversores, entidades públicas y privadas, legisladores e investigadores, entre otros.

\section{CONTENIDO DEL PROYECTO:}

La materia objeto del proyecto es la normativa contable. Entiéndese por tal toda dispocisión, ya sea de origen legal, oficial y/o profesional, que proponga una convención que -cualquiera que sea su nivel de obligatoriedad-arregle, condicione, oriente y/o establezca procedimientos, técnicas y/o actitudes que sobre la materia contable, la auditoría y la ética, deban respetar y aplicar los profesionales de la materia

En tal sentido el contenido del Código proyectado abarca los Principios de Contabilidad generalmente aceptados, las normas técnicas, resoluciones, postulados, guías de procedimientos, normas, declaraciones, informes, pronunciamientos y dictámenes emitidos por organismos profesionales, así como leyes, decretos y normas sancionados por poderes estatales. Se trata, por lo tanto, de todo tipo de prescripción positiva solo la contabilidad, la auditoría de cuentas y el ejercicio ético de la profesión.

Por las especiales características de un Código de esta naturaleza cuyas normas provienen de múltiples vertientes, se incluirá una brevísima referencia a la estructura jurídico-legal de cada uno de los países iberoamericanos, a fin de que el usuario conozca el proceso de sanción a normas de ese origen.

Se va a incluir igualmente el esquema de organización profesional reglamentado en cada país, puesto que las entidades profesionales no son fuente fundamental de normativa, sino que la agrupación colegiada la que otorga en la generalidad de los países el marco más adecuado para los sujetos concretos que aplican dicha normativa.

De esta forma, se entiende que el Código abarcará tanto la fuente de origen de las prescripciones contables en los países involucrados en el proyecto, como la descripción de las normas, su generación y el ámbito de su aplicación concreta.
El objetivo fundamental consiste, por lo tanto, en recopi lar, seleccionar, clasificar, sistematizar y desarrollar de forma descriptiva, las normas sobre contabilidad, auditoría y ética profesional vigentes en cada uno de los países de Iberoamérica a fin de:

- Posibilitar el conocimiento orgánico e integral sobre la si tuación concrela y particularizada de las normas vigentes en cada país.

- Documentar la práctica profesional vigente.

- Conformar una fuente documental que pueda servir de guía a empresas y profesionales en relación con temas supranacionales de contenido concreto.

- Servir de referencia para el futuro proceso de toma de decisiones respecto de la armonización del espacio económico iberoamericano.

- Establecer una base de datos de consulta sobre la problemática contable, de la auditoría y del ejercicio profesional, utilizable por empresas, organismos internacionales y nacionales, así como para académicos e investigadores.

- Conocer las distintas alternativas técnicas en uso frente a circunstancias concretas tales como: nuevos instrumentos de financiación, ambientes inflacionarios, agrupaciones empresriales, acuerdos comerciales, contenidos informativos internacionales, etc.

\section{AMBITO GEOGRAFICO Y ESTRUCTURA DEL PROYECTO AMBITO GEOGRAFICO:}

En la orientación de este proyecto se entıende por IBEROAMERICA, al conjunto de países hispano y lusoparlantes, geográficamente ubicados en Europa y América, que muestran unas raíces y una perspectiva histórica comunes que ya alcanza los 500 años.

Concrctamente los países incluídos en este Proyecto son:

AMERICA DEL SUR: ARGENTINA, CHILE, URUGUAY, PARAGUAY, BOLIVIA, BRASIL, PERU, ECUADOR, COLOMBIA, VENEZUELA.

CENTROAMERICA: PANAMA, HONDURAS. COSTA RICA, EL SALVADOR, NICARAGUA, MEXICO, CUBA, REPUBLICA DOMINICANA, PUERTO RICO.

$$
\text { EUROPA: ESPAÑA, PORTUGAL. }
$$

\section{IDIOMA:}

El proyecto se desarrollará en lengua castellana, excepto para la normativa vigente en Portugal y Brasil en que será bilingüe (castellano y portugués).

\section{ESTRUCTURA:}

La estructura básića del contenido del Proyecto es la siguiente:

A. Reseña sobre la organización jurídica de cada país.

B. Esquema de la Organización Profesional de los Contadores, Economistas y Auditores.

C. Jerarquía formal de las normas contables, de auditoría y éticas. 
C1. Normas de origen legal.

C2. Normas de origen profesional.

D. Proceso de sanción de las normas.

E. Normas Contables vigentes.

F. Normas de Auditoría vigentes.

G. Normas éticas vigentes.

H. Indice temático por país.

I. Indice temático general (por materias y por países).

\section{METODO DE ACTUALIZACION DE LA OBRA:}

Por las características y constante progreso de las materias contenidas en el Código se va a realizar, en primer lugar, la publicación del cuerpo de normas básicas, que se incluirá en una serie de volúmenes editados con un carácter integral a partir de aquí, se llevará a cabo una actualización recopilativa periódica de los datos, que se irá recogiendo en los correspondientes boletines o separatas de actualización.

\section{DIRECTORES DEL PROYECTO}

POR EUROPA: Dr. JESUS LIZCANO ALVAREZ. Catedrático de Economía Financiera y Contabilidad en la Universidad Autónoma de Madrid, y Vicesecretario General de la Asociación Española de Contabilidad y Administración de Empresas (AECA).

POR AMERICA: Dr. JORGE MANUEL GIL. Contador Público. (Federación Argentina de Consejos Profesionales en Ciencias Económicas). Director de la Revista TEUKEN y Profesor de la Universidad Nacional de la Patagonia San Juan Bosco.

\section{INSTITUCIONES ASESORAS NACIONALES}

Entre otras, han venido asesorando e informando sobre sus respectivos países, las siguientes entidades:

- Colegio de Contadores Públicos Autorizados de Puerto Rico.

- Corporación de Contadores de El Salvador.

- Instituto Mexicano de Contadores Públicos.

- Facultad de Contaduría y Administración de la Universidad Autónoma de México.

- Colegio de Contadores Públicos de Nicaragua.

- Facultad de Ciencias Económicas de la Universidad de La Habana (Cuba).

- Conselho Regional de Contabilidade do Rio Grande do Sul (Brasil).

- Colegio de Contadores Públicos Autorizados de Panamá.

- Facultad de Administración y Contaduría de la Universidad de Santiago de Chile.

- Colegio de Peritos Mercantiles y Contadores Públicos de Honduras.

- Instituto Brasileiro de Contadores Públicos.

- Colegio de Doctores de Ciencias Económicas y Contadores de Uruguay.

- Instituto Guatemalteco de Contadores Públicos y Auditores.

- Colegio de Profesionales en Ciencias Económicas de
Bolivia.

- Federación de Contadores Públicos de Venezuela.

- Facultad de Ciencias Contables de la Universidad de Cauca (Colombia).

- Colegio Colombiano de Contadores Públicos.

- Instituto Nacional de Contadores Públicos de Colombia.

- Departamento de Contaduría. Universidad de Antioquía (Colombia).

- Colegio de Contadores del Guayas (de la Federación Nacional de Contadores del Ecuador).

- Colegio de Contadores Públicos de Costa Rica).

- Sistema Nacional de Contabilidad de la República del Perú.

- Federación Argentina de Consejos Profesionales en Ciencias Económicas.

- Facultad de Ciencias Económicas (sede Comodoro Rivadavia) de la Universidad Nacional de La Patagonia San Juan Bosco.

- Asociación Española de Contabilidad y Administración de Empresas.

- Departamento de Contabilidad y Finanzas. Facultad de Economía. Universidad de Oriente (Cuba).

- Instituto de Pesquisa Augusto Tomelin (Brasil).

- Asociación Interamericana de Contabilidad (A.I.C.).

\section{USUARIOS Y DESTINATARIOS DEL PROYEC- TO}

Se prevé que el presente proyecto podrá ser utilizado por los siguientes usuarios, de los que también se estiman a priori algunas áreas de interés específicas:

\section{USUARIO: EMPRESAS EN GENERAL.}

\section{AREAS DE INTERES:}

- Requisitos formales del sistema contable vigente en cada país.

- Modelo contable utilizado (en el caso, Plan de Contabilidad).

- Obligatoriedad, oportunidad y alcance de la auditoría contable a la que debe someterse la información destinada a ser presentada a terceros.

- Organización, esquema y contenido de los estados contables utilizados en el país.

- Comprensión e interpretación de cuentas anuales de las empresas vinculadas jurídica, financiera y comercialmente.

- Marco institucional, jurídico, profesional vigente relativo a la contabilidad y la auditoría.

\section{USUARIO: PROFESIONALES DE LA CONTABILI- DAD Y AUDITORIA}

\section{AREAS DE INTERES:}

- Conocimientos concretos de desarrollo y actividad profesional de cada país.

- Incumbencias y alcances de la talla profesional así como 
requisitos personales requeridos para su desarrollo (nivel de titulación, experiencias, etc.).

- Normas y procedimientos de auditoría vigentes, oportunidad y responsabilidad profesionales.

- Código de ética sobre ejercicio profesional y campo de actuación específica.

- Aspectos específicos y particulares de cada país, en lo referente a la sistematización, confección, censura de cuentas, análisis e interpretación de la información contable;

- Técnicas de consolidación vigentes, tratamiento de las diferencias de cambio, ajustes e interpretación de resultados.

- Significado e interpretación del vocabulario.

\section{USUARIO: ORGANIZACIONES COLEGIADAS DE PROFESIONALES}

\section{AREAS DE INTERES:}

- Estudios comparativos sobre aspectos concretos de la organización y gestión profesional (matrículas, exámenes, responsabilidades, emisión de normas, estructura de honorarios, incumbencias profesionales, defensa y ética de la profesión, etc.).

- Referencias internacionales sobre tratamiento técnico de temas específicos.

- Posibilidad de establecer bases para armonizar técnicas y procedimientos que permitan homogenizar los contendos informativos y servir de base para un común lenguaje de los negocios.

- Formar parte activa en la negociación de convenios económicos bilaterales o multilaterales entre países iberoamericanos.

- Mantener un seguimiento actualizado sobre la evolución de la normativa en Iberoamérica.

\section{USUARIO: FACULTADES DE CIENCIAS ECONO- MICAS Y EMPRESRIALES}

\section{AREAS DE INTERES:}

- Bibliografía mínima, clasificada y compilada, sobre aspectos normativos en un importante área de la Contabilidad Internacional.

- Fuente documental básica para cualquier proyecto de tesis doctoral e investigación sobre normativa contable.

- Mantener una visión actualizada de la evolución de las normas a fin de ir introduciendo o modificando eventuales temáticas de estudio en sus planes académicos.

- Relacionar las exigencias profesionales, reales y formales, con el contenido y desarrollo de los planes de estudio.

\section{USUARIO: ASOCIACIONES EMPRESARIALES.}

\section{AREAS DE INTERES:}

- Conocer la estructura profesional de las ciencias empresariales, como aspecto concreto y referencial del sistema económico del país.

- Asesorar a las empresas asociadas en referencia a las materias del presente Código.

- Reforzar argumentos y sustentar peticiones a las autoridades gubernamentales, sobre la base de las experiencias internacionales referidas al sistema contable y la auditoría.

Establecer comparaciones sobre los modelos profesionales y contables vigentes, a fin de determinar relaciones de coste-utilidad de la información contable y la auditoría de las empresas.

\section{USUARIO: ENTIDADES GUBERNAMENTALES.}

\section{AREAS DE INTERES:}

- Carácter de la organización colegiada como fuente de poder institucional y de opinión profesional.

Modelos de control público sobre aspectos organizativos, técnicos y éticos de la profesión contable y de auditoría.

- Proceso de sanción de normas contables, así como sus características y eventual incidencia sobre el medio ambiente económico.

\section{METODOLOGIA DEL PROYECTO}

\section{CARACTERISTCAS DEL TRABAJO:}

A continuación se desarrollan las características específicas de cada etapa del proyecto a fin de su posterior cronograma. Se indica el contenido de cada una de las tareas concretas y cuál es su objetivo particular.

$1^{2}$ Etapa: RECOPILACION (proceso de búsqueda de la normativa contable a través de organismos profesionales colegiados, Facultades de Ciencias Económicas y Empresariales de Universidades del área geográfica, y académicos y profesionales colaboradores.

Objetivo: Acumular la normativa vigente en cada uno de los países.

2- Etapa: SELECCION (lectura y examen de la normativa acumulada a fin de detectar eventuales insuficiencias en la recopilación, iniciando las acciones para completar el archivo y descartando aquellas normas que no se vinculen con la materia del código).

Objetivo: Disponer de la normativa completa y pertinente al proyecto.

30 Etapa: CLASIFICACION PRIMARIA (agrupamiento e integración de las normas por país).

CLASIFICACION SECUNDARIA (agrupamiento por su origen: convencionales-las que provienen de la estructura profesional colegiada-, y legales -las que se derivan de leyes, decretos u ordenanzas gubernamentales).

Objetivo: Distinguir la normativa nacional, identificando su origen.

$4^{2}$ Etapa: SISTEMATIZACION (agrupación de las partidas por grupos temáticos).

Objetivo: Distribuir la normativa de cada país en base a la categorización y taxonomía adoptadas. 
5o Etapa; ELABORACION DE UN INDICE POR PAIS (sobre la sistematizacion temática).

LA ELABORACION DE UN INDICE TEMATICO (de cara a la posible búsqueda de alguna materia o tema determinado de contabilidad y auditoría, y su tratamiento en los diferentes países).

Objetivo: Culminar la preparación del proyecto y hacer operativo manejable de forma automática el amplio contenido de la obra.

Etapa final: PUBLICACION Y DIFUSION DEL "CODIGO IBEROAMERICANO DE CONTABILIDAD Y AUDITORIA".

Objetivo: Ofrecer una obra recopilativa sobre contabilidad y auditoría en el mundo iberoamericano, que sirva de homenaje permanente a partir de 1992 para esta comunidad histórica, y sea de utilidad para todos los sectores y colectivos relacionados de una u otra forma con la contabilidad y la auditoría a nivel internacional.

\section{Anexo IV}

\section{DE LOS OBJETIVOS}

ARTICULO 6.- Los objetivos fundamentales de la confederación son:

a) El fomento de la solidaridad entre todos los Contadores Públicos Ibeoamericanos.

b) Mantener sus actividades en permanente dirección, hacia el análisis, e investigación y orientación de los problemas que, en materia de contabilidad, auditoría y demás disciplinas afines y/o conexas surjan en la economía de los Países Miembros.

c) Velar por el respeto del Contador Público en el ejercicio de su profesión.

d) Tender a que el Contador Público adquiera una adecuada conciencia profesional y social acorde con su participación integral en el desarrollo de la comunidad.

e) Crear en los países miembros un sentido claro y definido sobre el proceso de desarrollo económico y la integración, de todos los órdenes de la convivencia humana, para el logro de justos y equitativos beneficios para la comunidad.

f) Fomentar la investigación y divulgación de las ciencias contables a objeto de mantener activo el espíritu creador del Contador Público.

g) Contribuir a la consecución y promulgación de leyes de ejercicio-profesional, que favorezcan las actividades del Contador Público y vigilar su correcta aplicación.

h) Propugnar porque las funciones de Contadores, Auditores Internos y Externos, Comisarios, Revisores, Fiscales, Peritos, Contables y demás competencia del Contador Público, solo serán ejercida por profesionales legalmente autorizados por los organismos rectores de la profesión, en cada País Miembro.

i) Luchar por defender los intereses del profesional Contador Público nacional, de cada uno de los países miembros, contra los intereses de personas o firmas nacionales o transnacionales de Contadores Públicos que atenten contra ellos.

j) Propugnar porque en los miembros, los organismos de funcionamiento y fomento regionales y nacionales, reconozcan en sus respectivas actividades y operaciones, la actuación del Contador Público; tanto en la fase de evaluación de los proyectos, como en la administración, control y auditoría independientes de los prestatarios eventuales y programas o proyectos financieros.

k) Propiciar el intercambio del ejecicio profesional entre los Contadores Públicos Nacionales. Miembros de los Gremios representados en la Confederación.

1) Sostener medios permanentes de información y comunicación iberoamericana del desarrollo de la Contaduría Pública.

m) Contribuir a la unificación de lineamientos éticos del Contador Público.

n) Tener vinculación estrecha con la Universidad y colaborar en la creación de planes y programas de estudios curricular, acorde a las necesidades de cada país.

ñ) Afiliar a todas aquellas organizaciones internacionales que tengan relación con la profesión, que se crean convenientes para el robustecimiento institucional.

o) Propender por la protección social del Contador Público de sus familiares de cada país miembro.

p) Emitir y publicar pronunciamientos técnicos en materia de Contaduría Pública, con el carácter de recomendaciones a los países miembros, con las adaptaciones lógicas según las leyes de cada país.

q) Establecer comunicación y relaciones permanentes con organismos interancionales mundiales y regionales. A objeto de que el Contador Público se proyecte en distintas direcciones.

r) Los países miembros de esta Confederación, deberán o podrán formular planteamientos de fondo sobre las políticas contables al sector gubernamental, a fin de proteger el patrimonio nacional en todas sus áreas.

s) Estudiar la problemática multilateral, relacionada con el orden financiero, económico, político y social; de lo cual se harán los pronunciamientos.

t) Los organismos miembros de esta Confederación, deben emitir sus opiniones a las autoridades competentes en cada país, en las áreas de su especialización, como aporte al desarrollo de la sociedad.

u) Propender para que cada país se aprueben las leyes de carácter nacionalista, que impidan el ejercicio de las firmas transnacionales de contabilidad y auditoría.

v) Cualquier otro objetivo que tienda a robustecer los lazos de amistad, a los fines de la Confederación. 